ÊESTI NSV TEADUSTE AKADEEMIA TOIMETISED. 31. KOIDE FOOSIKA * MATEMAATIKA. 1982, NR. 1

ИЗВЕСТИЯ АКАДЕМИИ НАУК ЭСТОНСКОИ ССР. ТОМ З1 ФИЗИКА * МАТЕМАТИКА. 1982, № 1

\title{
МЕТОД ДИСКРЕТНЫХ ОРДИНАТ ПРИМЕНИТЕЛЬНО К РЕШЕНИЮ УРАВНЕНИЯ ПЕРЕНОСА ИЗЛУЧЕНИЯ В ОДНОРОДНОЙ ПЛОСКОПАРАЛЛЕЛЬНОЙ АНИЗОТРОПНО РАССЕИ ВАЮЩЕЙ АТМОСФЕРЕ. 1
}

(Представил Г. Кузмин)

Метод дискретных ординат основан на замене интегрального члена уравнения переноса излучения какой-либо квадратурной формулой. Благодаря этому интегро-дифференциальное уравнение переноса излучения приближенно заменяется системой линейных дифференциальных уравнений с постоянными коэффициентами. В [ $\left.{ }^{1-3}\right]$ этот метод исследован для решения уравнения переноса излучения при изотропном рассеянии. При решении методом дискретных ординат задачи об анизотропном рассеянии система дифференциальных уравнений имеет большую размерность. В настоящей работе показано, что при специальном выборе квадратурных формул матрица коэффициентов системы дифференциальных уравнений естественным образом разбивается на блоки, которые коммутируют между собой, легко приводятся при помощи унитарного преобразования, не зависящего от конкретного блока, к диагональному виду и, следовательно, легко обращаются.

\section{1. Дискретизация уравнения переноса излучения}

Рассмотрим интегро-дифференциальное уравнение переноса излучения в однородной анизотропной плоскопараллельной атмосфере, освещенной параллельными солнечными лучами [ $\left.{ }^{4}\right]$

$$
\begin{aligned}
\mu \frac{d I(\tau, \mu, \varphi)}{d \tau}= & -I(\tau, \mu, \varphi)+\frac{\lambda}{4 \pi} \int_{0}^{2 \pi} \int_{-1}^{1} g(\gamma) I\left(\tau, \mu^{\prime}, \varphi^{\prime}\right) d \varphi^{\prime} d \mu^{\prime}+ \\
& +\frac{\lambda c_{0}}{4 \pi} g\left(\gamma^{\prime}\right) \exp (\sigma \tau), \\
I(0, \mu, \varphi)=0 & (0<\mu \leqslant 1), \quad I(H, \mu, \varphi)=0 \quad(-1 \leqslant \mu<0) .
\end{aligned}
$$

Здесь $\lambda$ - вероятность выживания фотона при элементарном акте рассеяния; $c_{0}=\pi S-$ солнечная постоянная; $H-$ оптическая толщина атмосферы; $g(\gamma) \geqslant 0-$ индикатриса рассеяния, нормированная условием

$$
\begin{gathered}
(4 \pi)^{-1} \int_{\omega} g(\gamma) d \omega=(4 \pi)^{-1} \int_{0}^{2 \pi} \int_{-1}^{1} g(\gamma) d \varphi d \mu=1, \quad \gamma \in[0, \pi], \\
\cos (\gamma)=\mu \mu^{\prime}+\sqrt{\left(1-\mu^{2}\right)\left(1-\mu^{\prime 2}\right)} \cos \left(\varphi-\varphi^{\prime}\right),
\end{gathered}
$$




$$
\cos \left(\gamma^{\prime}\right)=\mu \mu_{0}+\sqrt{\left(1-\mu^{2}\right)\left(1-\mu_{0}^{2}\right)} \cos \left(\varphi-\varphi_{0}\right),
$$

$\left(-\arccos \mu_{0}, \varphi_{0}\right)$ - направление солнечных лучей в сферической системе координат с полярной осью, идущей в сторону растущих оптических глубин; $\sigma=-1 / \mu_{0}$. Решение этого уравнения $I(\tau, \mu, \varphi)$ дает интенсивность многократно рассеянного солнечного излучения на оптической глубине $\tau$ в направлении, которое определяется полярным расстоянием $\arccos \mu$ и азимутом $\varphi$.

Заменим интегральный член в уравнении (1) некоторыми квадратурными формулами

$$
\begin{gathered}
\int_{0}^{2 \pi} \xi(\varphi) d \varphi \approx \sum_{m=1}^{p} \beta_{m} \xi\left(\varphi_{m}\right), 0<\varphi_{1}<\ldots<\varphi_{p} \leqslant 2 \pi, \sum_{m=1}^{p} \beta_{m}=2 \pi \\
\int_{-1}^{1} \zeta(\mu) d \mu \approx \sum_{i=1}^{n} \alpha_{i}\left(\zeta\left(\mu_{i}\right)+\zeta\left(-\mu_{i}\right)\right), 0<\mu_{1}<\ldots<\mu_{n} \leqslant 1, \sum_{i=1}^{n} \alpha_{i}=1 .
\end{gathered}
$$

Отсюда интегральное уравнение (1) заменяется системой линейных дифференциальных уравнений

$$
\begin{gathered}
\mu_{s} \frac{d I_{s m}(\tau)}{d \tau}=-I_{s m}(\tau)+\frac{\lambda}{4 \pi} \sum_{i=1}^{n} \sum_{l=1}^{p} g_{i l s m} I_{i l}(\tau) \alpha_{i} \beta_{l}+ \\
+\frac{\lambda}{4 \pi} \sum_{i=1}^{n} \sum_{l=1}^{p} g_{-i l s m} I_{-i l}(\tau) \alpha_{i} \beta_{l}+\exp (\sigma \tau) u_{s m} \\
s= \pm 1, \pm 2, \ldots, \pm n ; m=1,2, \ldots, p ; \mu_{-i}=-\mu_{i}
\end{gathered}
$$

в которой, учитывая (4), мы положим

$$
\begin{gathered}
g_{ \pm i l s m^{\prime}}=g\left(\gamma_{ \pm i l s m}\right), \quad u_{s m}=\lambda c_{0} g\left(\gamma_{s m}^{\prime}\right) / 4 \pi, \\
\cos \left(\gamma_{ \pm i l s m}\right)= \pm \mu_{s} \mu_{i}+\sqrt{\left(1-\mu_{s}^{2}\right)\left(1-\mu_{i}^{2}\right)} \cos \left(\varphi_{m}-\varphi_{l}\right), \\
\cos \left(\gamma_{s m}^{\prime}\right)=\mu_{s} \mu_{0}+\sqrt{\left(1-\mu_{s}^{2}\right)\left(1-\mu_{0}^{2}\right)} \cos \left(\varphi_{m}-\varphi_{0}\right) ; \quad \gamma_{i l s m}, \gamma_{s m}^{\prime} \in[0, \pi] .
\end{gathered}
$$

Согласно (2), запишем краевые условия для системы дифференциальных уравнений (6):

$$
I_{i m}(0)=I_{-i m}(H)=0, \quad i=1,2, \ldots, n ; m=1,2, \ldots, p .
$$

Делая в случае необходимости перенормировку, положим

$$
\begin{aligned}
& (4 \pi)^{-1} \sum_{i=1}^{n} \sum_{l=1}^{p}\left(g_{i l s m}+g_{-i l s m}\right) \alpha_{i} \beta_{l}=1, \\
& s= \pm 1, \pm 2, \ldots, \pm n ; \quad m=1,2, \ldots, p .
\end{aligned}
$$

Последнее выражение является по существу дискретным аналогом условия (3).

В настоящей работе будем исследовать систему дифференциальных уравнений (6) с условиями (7) и (8).

\section{2. Существование и единственность решения дискретизированного уравнения переноса излучения}

Для доказательства существования решения дискретизированного уравнения переноса излучения преобразуем задачу (6)-(8) в интегральное 
уравнение III рода. K полученному уравнению применим теорему Банаха о единственности решения операторных уравнений II рода. Положим

$$
y_{s m}(\tau)=(\lambda / 4 \pi) \sum_{i=1}^{n} \sum_{l=1}^{p}\left(g_{i l s m} I_{i l}(\tau)+g_{-i l s m} I_{-i l}(\tau)\right) \alpha_{i} \beta_{l} .
$$

Решая систему дифференциальных уравнений (6) относительно $I_{s m}(\tau)$, получим

$$
\begin{aligned}
I_{i m}(\tau) & =\int_{0}^{\tau} y_{i m}\left(\tau^{\prime}\right) \exp \left(-\left(\tau-\tau^{\prime}\right) / \mu_{i}\right) \frac{d \tau^{\prime}}{\mu_{i}} \\
i & =1,2, \ldots, n ; \quad m=1,2, \ldots, p
\end{aligned}
$$

$I_{i m}(\tau)=-\int_{\tau}^{H} y_{i m}\left(\tau^{\prime}\right) \exp \left(-\left(\tau-\tau^{\prime}\right) / \mu_{i}\right) \frac{d \tau^{\prime}}{\mu_{i}}, \quad i=-1,-2, \ldots,-n$.

Подставив найденное выражение в (9), получим систему интегральных уравнений

$$
\begin{aligned}
y_{s m}(\tau) & =\int_{0}^{\tau} \sum_{i=1}^{n} \sum_{l=1}^{p}\left(\lambda \alpha_{i} \beta_{l}\left(4 \pi \mu_{i}\right)^{-1} g_{i l s m}\right) \exp \left(-\left(\tau-\tau^{\prime}\right) / \mu_{i}\right) y_{i l}\left(\tau^{\prime}\right) d \tau^{\prime}+ \\
& +\int_{\tau}^{H} \sum_{i=1}^{n} \sum_{l=1}^{p}\left(\lambda \alpha_{i} \beta_{l}\left(4 \pi \mu_{i}\right)^{-1} g_{-i l s m}\right) \exp \left(\left(\tau-\tau^{\prime}\right) / \mu_{i}\right) y_{-i l}\left(\tau^{\prime}\right) d \tau^{\prime}+ \\
& +\exp (\sigma \tau) u_{s m}, \quad s= \pm 1, \pm 2, \ldots, \pm n ; \quad m=1,2, \ldots, p .
\end{aligned}
$$

Отметим, что задача $(6)-(8)$ и система (11) эквивалентны в төом смысле, что если функции $I_{s m}(\tau)$ являются решением задачи $(6)-(8)$, то $y_{s m}(\tau)$, определенные выражением (9), удовлетворяют системе (11) и, наоборот, если $y_{s m}(\tau)$ являгтся решением системы $(11)$, то функции $I_{s m}(\tau)$, определенные формулами $(10)$, являются решением задачи (6) $-(8)$.

Рассмотрим систему интегральных уравнений (11) как операторное уравнение II рода в пространстве с нормой

$$
\|y\|=\max _{1 \leqslant m \leqslant p} \max _{\substack{-n \leqslant s \leqslant n \\ s \neq 0}} \max _{0 \leqslant \tau \leqslant H}\left|y_{s m}(\tau)\right| .
$$

Тогда норма оператора $K$, где сумма интегральных членов в выражении (11) описывается символом $K y$, удовлетворяет неравенству [ $\left.{ }^{5}\right]$

$$
\|K\|<\lambda \leqslant 1 \text {. }
$$

Отсюда система интегральных уравнений (11), а следовательно, и задача (6)-(8) имеют единственное решение.

\section{3. Матричная запись дискретизированного уравнения переноса излучения}

Пусть $I_{n}-$ единичная матрица размерности $n \times n, e_{j}^{n}-$ вектор-столбец длины $n$, у которого на $j$-м месте стоит единица, а на остальных нули; $\otimes-$ символ кронекеровского произведения матриц ([ $\left.{ }^{6}\right]$, с. 259$)$; $\delta_{i j}-$ символ Кронекера; $\left(c_{i j}\right)_{i j}$ - матрица с элементами * $c_{i j}$; $\left(c_{j}\right)_{j}$ - вектор-столбец с элементами $c_{j}$. Положим**

* $i$-я строка этой матрицы есть $\left(c_{i 1} c_{i 2} \ldots c_{i n}\right)$

** Ниже подразумевается следующее изменение индексов: $i, j=1,2, \ldots, n ; k, s=$ $= \pm 1, \pm 2, \ldots, \pm n ; m, l=1,2, \ldots, p$. 


$$
\alpha=\left((\lambda / 2) \alpha_{i} \dot{\delta}_{i j}\right)_{i j}, \quad \gamma_{m t}=\dot{\beta}_{m} / 2 \pi, \quad \Gamma=\left(\delta_{m l} \gamma_{l}\right)_{m l},
$$

$\Pi=I_{2} \otimes \alpha \otimes \Gamma, \quad W_{k s}=\left(g_{s l k m}\right)_{m l}, \quad V_{1}=\left(W_{i j}\right)_{i j}, \quad V_{2}=\left(W_{-i j}\right)_{i j}$,

$$
\begin{gathered}
W=\left(\begin{array}{ll}
V_{1} & V_{2} \\
V_{2} & V_{1}
\end{array}\right), \quad E=\left(\begin{array}{cc}
I_{n p} & 0 \\
0 & -I_{n p}
\end{array}\right), \quad b=\left(\delta_{i j} b_{j}\right)_{i j}, \\
\mathrm{~B}=I_{2} \otimes b \otimes I_{p}, \quad I_{s}(\tau)=\left(I_{s m}(\tau)\right)_{m}, \quad u_{s}=\left(u_{s m}\right)_{m}, \\
x(\tau)=\left(I_{i}(\tau)\right)_{i}, \quad y(\tau)=\left(I_{-i}(\tau)\right)_{i}, \quad f_{1}=\left(u_{i}\right)_{i}, \quad f_{2}=\left(u_{-i}\right)_{i}, \\
v(\tau)=e_{1}^{2} \otimes x(\tau)+e_{2}^{2} \otimes y(\tau), \quad f=e_{1}^{2} \otimes f_{1}+e_{2}^{2} \otimes f_{2} .
\end{gathered}
$$

Отметим некоторые свойства $g_{\text {ilsm }}$, которые следуют из определения индикатрисы рассеяния (4)

$$
\begin{gathered}
g_{i l s m}=g_{-i l(-s) m}, \quad g_{-i l s m}=g_{i l(-s) m}, \\
g_{i l s m}=g_{s l i m}=g_{s m i l} .
\end{gathered}
$$

Учитывая эти свойства и введенные в этом разделе обозначения, запишем задачу $(6)-(8)$ в виде

$$
\begin{array}{r}
\mu_{s} \frac{d I_{s}(\tau)}{d \tau}=-I_{s}(\tau)+\sum_{j=1}^{n} W_{s j}\left(\lambda \alpha_{j} / 2\right) \Gamma I_{j}(\tau)+ \\
+\sum_{j=1}^{n} W_{-s j}\left(\lambda \alpha_{j} / 2\right) \Gamma I_{-j}(\tau)+\exp (\sigma \tau) u_{s}, \\
s= \pm 1, \pm 2, \ldots, \pm n, \\
I_{j}(0)=I_{-j}(H)=0, \quad j=1,2, \ldots, n .
\end{array}
$$

Умножив $s$-ю строку этой системы на $b_{s}=1 / \mu_{s}$, получим

$$
\begin{gathered}
x^{\prime}(\tau)=\mathcal{B}\left(V_{1} \Pi_{1}-I_{n p}\right) x(\tau)+\mathcal{B} V_{2} \Pi_{1} y(\tau)+\exp (\sigma \tau) \mathcal{B} f_{1}, \\
y^{\prime}(\tau)=-\mathcal{B}\left(V_{1} \Pi_{1}-I_{n p}\right) y(\tau)-\mathcal{B} V_{2} \Pi_{1} x(\tau)-\exp (\sigma \tau) \mathcal{B} f_{2}, \\
x(0)=y(H)=0,
\end{gathered}
$$

где $\mathcal{B}=b \otimes I_{p}, \Pi_{1}=\alpha \otimes \Gamma$.

Кроме того, будем использовать еще одну запись задачи $(6)-(8)$ :

$$
\begin{gathered}
v^{\prime}=R v+\mathrm{B} E f \exp (\sigma \tau), \quad R=\mathrm{B} E\left(W \Pi-I_{2 n p}\right), \\
x(0)=y(H)=0 .
\end{gathered}
$$

\section{4. Собственные значения и собственные векторы матрицы коэффициентов системы дифференциальных уравнений (16)}

Рассмотрим случай $0 \leqslant \lambda<1$.

Те ор ем а. Матрица $R=\mathrm{B} E\left(W \Pi-I_{2 n p}\right)$ обладает следующими свойствами:

1. Собственные значения матрицы $R$ вещественны;

2. Матрица $R$ подобна диагональной матрище, состоящей из собственных значений матрицы $R$;

3. Если и есть собственное значение матрицы $R$, то - $\mu$ является также собственным значением матрищы $R$;

4. Если $z=e_{1}{ }^{2} \otimes \vartheta_{1}+e_{2}{ }^{2} \otimes \vartheta_{2}$ есть собственный вектор матрицы $R$, 
то $\hat{z}=e_{1}^{2} \otimes \vartheta_{2}+e_{2}^{2} \otimes \vartheta_{1}$ также является собственным вектором матрицы $R\left(\vartheta_{1}, \vartheta_{2} \in R^{n p}\right)$.

Для доказательства первых двух свойств нам потребуется следующая Л емм а. Всякое собственное значение $\mu$ матрицы $\left(W \Pi-I_{2 n p}\right)$ удовлетворяет неравенству

$$
-\lambda-1 \leqslant \mu \leqslant \lambda-1
$$

Доказ ательство леммы. Из (13) следует, что матрица W симметрична. Далее, матрица WI является самосопряженным оператором в гильбертовом пространстве со скалярным произведением

$$
(x, y)_{\Pi}=(\Pi x, y) .
$$

Отсюда, по теореме Данфорда ([ $\left.{ }^{7}\right]$, с. 456), спектр матрицы $\left(W \Pi-I_{2 n p}\right)$ есть

$$
\{\mu-1 \mid \mu \in \text { спектр }(W \Pi)\} .
$$

Собственные значения $\eta$ матрицы $W \Pi$ удовлетворяют неравенству

$$
|\eta| \leqslant\|W \Pi\|_{1}
$$

где $\|\cdot\|_{1}-$ некоторая норма. Если положим

$$
\|A\|_{1}=\max _{i} \sum_{j}\left|a_{i j}\right|, \quad A=\left(a_{i j}\right)_{i j},
$$

то, учитывая (8) и то, что $g(\gamma) \geqslant 0$, получим

$$
|\eta|=\max _{\substack{-n \leqslant s \leqslant n \\ s \neq 0}} \max _{\substack{1 \leqslant m \leqslant p \\ 1 \leqslant m}}(\lambda / 4 \pi) \sum_{i=1}^{n} \sum_{l=1}^{p}\left(g_{\text {ilsm }}+g_{-i l s m}\right) \alpha_{i} \beta_{l}=\lambda .
$$

Последнее доказывает лемму.

Дока $з$ а тельст во т е о ремы. При $0 \leqslant \lambda<1$ матрицу (WП $-I_{2 n p}$ ) можно рассматривать как отрицательно определенный самосопряженный оператор в гильбертовом пространстве со скалярным произведением (18). Отсюда и матрица $R$ является самосопряженным оператором в гильбертовом пространстве со скалярным произведением

$$
\langle x, y\rangle=-\left(\left(W \Pi-I_{2 n p}\right) x, y\right)_{\Pi} .
$$

Значит, спектр матрицы $R$ вещественный. Кроме того, собственные векторы матрицы $R$ образуют полную ортонормированную систему в пространстве со скалярным произведением (19) ([7], с. 252). Следовательно, имеет место свойство 2.

Докажем теперь последние два свойства. Пусть $z=e_{1}^{2} \otimes \vartheta_{1}+$ $+e_{2}^{2} \otimes \vartheta_{2}$ есть собственный вектор матрицы $R$, соответствующий собственному значению $\mu$. Тогда, учитывая (15), получим

$$
\begin{aligned}
& C \vartheta_{1}+D \vartheta_{2}=\mu \vartheta_{1}, \\
& -D \vartheta_{1}-C \vartheta_{2}=\mu \vartheta_{2}, \quad C=\Re\left(V_{1} \Pi_{1}-I_{n p}\right), \quad D=\mathcal{B}_{2} \Pi_{1} .
\end{aligned}
$$

Умножив эту систему на $(-1)$ и поменяв местами строчки, получим

$$
\begin{gathered}
C \vartheta_{2}+D \vartheta_{1}=(-\mu) \vartheta_{2}, \\
-D \vartheta_{2}-C \vartheta_{1}=(-\mu) \vartheta_{1} .
\end{gathered}
$$

Последнее завершает доказательство теоремы. 


\section{5. Выбор квадратурных формул. Исследование дискретизированного уравнения переноса излучения}

Пусть квадратурная формула (5) есть формула средних прямоугольников, т. е.

$$
\beta_{m}=2 \pi / p, \quad \varphi_{m}=2 \pi(m-1 / 2) / p, \quad m=1,2, \ldots, p .
$$

Рассмотрим случай четного $p$, т. е. $p=2 q$, и отметим, что все приводимые ниже результаты легко переносятся на нечетный $p$. Кроме того, с целью некоторых упрощений азимут прямого солнечного излучения $\varphi_{0}$ положим равным $\pi / 2 q$. С учетом этих предположений дискретизированное уравнение переноса излучения (14) примет вид

$$
\begin{gathered}
\frac{d I_{s}(\tau)}{d \tau}=\sum_{j=1}^{n} b_{s} W_{s j}\left(\lambda \alpha_{j} / 2\right) \frac{1}{p} I_{j}(\tau)-b_{s} I_{s}(\tau)+ \\
+\sum_{j=1}^{n} b_{s} W_{-s j}\left(\lambda \alpha_{j} / 2\right) \frac{1}{p} I_{-j}(\tau)+b_{s} \exp (\sigma \tau) u_{s}, \quad s= \pm 1, \pm 2, \ldots, \pm n \\
I_{j}(0)=I_{-j}(H)=0, \quad j=1,2, \ldots, n .
\end{gathered}
$$

Из (12) и (13) следует

$$
W_{s i}=W_{i s}, \quad W_{s i}^{\mathrm{T}}=W_{s i}, \quad W_{-s i}=W_{s(-i)}, \quad W_{(-i)(-s)}=W_{i s} .
$$

Согласно определению матрицы $W_{i j}(i, j=1,2, \ldots, n)$, имеем

$$
W_{i j}=\left(g_{j l i m}\right)_{m l}=\left(g\left(\gamma_{j l i m}\right)\right)_{m l}, \quad i, j=1,2, \ldots, n ; m, l=1,2, \ldots, p,
$$

где

$$
\cos \left(\gamma_{j l i m}\right)=\mu_{i} \mu_{j}+\sqrt{\left(1-\mu_{i}^{2}\right)} \overline{\left(1-\mu_{j}^{2}\right)} \cos (2 \pi(m-l) / p)=\cos \left(\gamma_{i j}^{|m-l|}\right) .
$$

Учитывая четность $p=2 q$, свойства функции $\cos x$ и то, что функция $g(\gamma)$ определена на $[0, \pi]$, получим, что $W_{i j}$ является циркулянтом ([6], с. 263), т. е. матрицей вида

$$
C=\left(\begin{array}{ccccc}
c_{0} & c_{1} & c_{2} & \ldots & c_{p-1} \\
c_{p-1} & c_{0} & c_{1} & \ldots & c_{p-2} \\
\cdot & \cdot & \cdot & \cdot & \cdot \\
c_{1} & c_{2} & c_{3} & \ldots & c_{0}
\end{array}\right)
$$

Упорядоченный набор $\left(c_{0} c_{1} c_{2} \ldots c_{p-1}\right)$ будем называть образующим вектором для циркулянта $C$. Образующий вектор для циркулянта $W_{i j}$ есть

$$
\left(x_{i j}^{0} x_{i j}^{1} \ldots x_{i j}^{q-1} x_{i j}^{q} x_{i j}^{q-1} \ldots x_{i j}^{1}\right), \quad i, j=1,2, \ldots, n
$$

где

$$
\begin{array}{r}
x_{i j}^{r}=g\left(\gamma_{i j}^{r}\right), \quad \cos \left(\gamma_{i j}^{r}\right)=\mu_{i} \mu_{j}+\sqrt{\left(1-\mu_{i}^{2}\right)\left(1-\mu_{j}^{2}\right)} \cos (r \pi / q), \\
r=0,1,2, \ldots, q .
\end{array}
$$

Для матрицы $W_{-i j}(i, j=1,2, \ldots, n)$ имеем

$$
\begin{gathered}
\cos \left(\gamma_{-j l i m}\right)=-\mu_{i} \mu_{j}+\sqrt{\left(1-\mu_{i}^{2}\right)\left(1-\mu_{j}^{2}\right)} \cos (\pi(m-l) / q)= \\
=-\left(\mu_{i} \mu_{j}+\sqrt{\left(1-\mu_{i}^{2}\right)\left(1-\mu_{j}^{2}\right)} \cos (\pi-(m-l) \pi / q)=-\cos \left(\gamma_{i j}^{q-|m-l|}\right) .\right.
\end{gathered}
$$


Отсюда следует то, что $\gamma_{-i j}^{r}=\pi-\gamma_{i j}^{q-r}$ и что образующий вектор для циркулянта $W_{-i j}$ есть

$$
\left(x_{-i j}^{0} x_{-i j}^{1} \ldots x_{-i j}^{q-1} x_{-i j}^{q} x_{-i j}^{q-1} \ldots x_{-i j}^{1}\right), \quad i, j=1,2, \ldots, n .
$$

Здесь $x_{-i j}^{r}=g\left(\pi-\gamma_{i j}^{q-r}\right), r=0,1,2, \ldots, q$.

Отметим некоторые свойства циркулянтов. Собственное значение циркулянта (22) определяется формулой $\left(\left[{ }^{6}\right]\right.$; с. 263$)$

$$
\lambda_{k}=c_{0}+\sum_{j=1}^{p-1} r_{k}^{j} c_{j},-
$$

а соответствующий ему собственный вектор есть

$$
\left(\begin{array}{lllll}
1 & r_{k} & r_{k}^{2} & \ldots & r_{k}^{p-1}
\end{array}\right)^{\mathrm{T}},
$$

где $r_{k}-k$-й корень уравнения $r^{p}=1$. Отсюда следует, что любой циркулянт при помощи унитарного преобразования

$$
U=\frac{1}{\sqrt{p}}\left|\begin{array}{cccc}
1 & 1 & \ldots & 1 \\
r_{1} & r_{2} & \ldots & 1 \\
\cdot & \cdot & \ldots & 1 \\
\cdot & . & \ldots & . \\
r_{1}^{p-1} & r_{2}^{p-1} & \ldots & 1
\end{array}\right|, \quad U U^{*}=I_{p}
$$

не зависящего от конкретного циркулянта, приводится к диагональному виду. Следовательно, матрицы $W_{k s}$ коммутируют между собой.

Применяя формулу (25) к циркулянту (23), получим в явном виде собственные значения $\lambda_{i j}{ }^{k}$ матрицы $W_{i j}(i, j=1,2, \ldots, n)$

$$
\begin{aligned}
\lambda_{i j}^{k} & =x_{i j}^{0}+x_{i j}^{q} r_{k}^{q}+\sum_{l=1}^{q-1} x_{i j}^{l}\left(r_{k}^{l}+r_{k}^{p-l}\right)= \\
& =x_{i j}^{0}+x_{i j}^{q}(\cos (2 \pi k q / p)+\mathbf{i} \sin (2 \pi k q / p))+ \\
& +\sum_{l=1}^{q-1} x_{i j}^{l}(\cos (2 \pi k l / p)+\mathbf{i} \sin (2 \pi k l / p)+\cos ((p-l) 2 \pi k / p)+ \\
& +\mathbf{i} \sin ((p-l) 2 \pi k / p))= \\
& =x_{i j}^{0}+(-1)^{k} \varkappa_{i j}^{q}+2 \sum_{l=1}^{q-1} x_{i j}^{l} \cos (\pi k l / q), \quad k=1,2, \ldots, p ; \quad \mathbf{i}=\sqrt{-1} .
\end{aligned}
$$

Аналогично, применяя формулу (25) к циркулянту (24), получим

$\lambda_{-i j}^{k}=x_{-i j}^{0}+(-1)^{k} \chi_{-i j}^{q}+2 \sum_{l=1}^{q-1} x_{-i j}^{l} \cos (\pi k l / q), \quad k=1,2, \ldots, p$.

Вычислим теперь вектор $U^{*} u_{s}$, где $U$ - матрица, определенная (26). По определению $u_{s}$ (см. раздел 3 ) имеем

$$
u_{s m}=\lambda c_{0} g\left(\gamma_{s m}^{\prime}\right) / 4 \pi, \quad s= \pm 1, \pm 2, \ldots, \pm n ; \quad m=1,2, \ldots, p,
$$

где

$$
\begin{gathered}
\cos \left(\gamma_{s m}^{\prime}\right)=\mu_{s} \mu_{0}+\sqrt{\left(1-\mu_{s}^{2}\right)\left(1-\mu_{0}^{2}\right)} \cos (2 \pi(m-1 / 2) / p-\pi /(2 q))= \\
=\mu_{s} \mu_{0}+\sqrt{\left(1-\mu_{s}^{2}\right)\left(1-\mu_{0}^{2}\right)} \cos (\pi(m-1) / q)=\cos \left(\gamma_{s 0}^{|m-1|}\right) .
\end{gathered}
$$


Последнее позволяет записать вектор $u_{s}$ в виде

$$
\begin{gathered}
u_{i}=\left(\lambda c_{0} / 4 \pi\right)\left(x_{i 0}^{0} x_{i 0}^{1} \ldots x_{i 0}^{q-1} x_{i 0}^{q} x_{i 0}^{q-1} \ldots x_{i 0}^{1}\right)^{\mathrm{T}}, \\
u_{-i}=\left(\lambda c_{0} / 4 \pi\right)\left(x_{-i 0}^{0} x_{-i 0}^{1} \cdots x_{-i 0}^{q-1} x_{-i 0}^{q} x_{-i 0}^{q-1} \ldots x_{-i 0}^{1}\right)^{\mathrm{T}}, \quad i=1,2, \ldots, n .
\end{gathered}
$$

Здесь $\quad x_{i 0}^{r}=g\left(\gamma_{i 0}^{r}\right), x_{-i 0}^{r}=g\left(\pi-\gamma_{i 0}^{q-r}\right)$. Отсюда, $m$-я координата $\left(U^{*} u_{s}\right)^{m}$ вектора $U^{*} u_{s}$ есть

$$
\begin{gathered}
\left(U^{*} u_{s}\right)^{m}=\left(\lambda c_{0} / 4 \pi \sqrt{p}\right)\left(x_{s 0}^{0}+x_{s 0}^{q} \bar{r}_{m}^{q}+\sum_{l=1}^{q-1} x_{s 0}^{l}\left(\bar{r}_{m}^{l}+\bar{r}_{m}^{p-l}\right)\right)= \\
=\left(\lambda c_{0} / 4 \pi \sqrt{p}\right)\left(x_{s 0}^{0}+(-1)^{m} x_{s 0}^{q}+2 \sum_{l=1}^{q-1} x_{s 0}^{l} \cos (\pi m l / q)\right) \\
s= \pm 1, \pm 2, \ldots, \pm n ; \quad m=1,2, \ldots, p .
\end{gathered}
$$

Умножив слева систему (20) на матрицу $U^{*}$ и учитывая (21), (27)(29), получаем задачу (20) в виде

$$
\begin{gathered}
\frac{d \hat{I}_{s}(\tau)}{d \tau}=\sum_{j=1}^{n} \Lambda(s, j) \hat{I}_{j}(\tau)-b_{s} \hat{I}_{s}(\tau)+ \\
+\sum_{j=1}^{n}(-\Lambda(-s, j)) \hat{I}_{-j}(\tau)+\hat{u}_{s} \exp (\sigma \tau), \quad s= \pm 1, \ldots, \pm n, \\
\hat{I}_{j}(0)=\hat{I}_{-j}(H)=0, \quad j=1,2, \ldots, n,
\end{gathered}
$$

где $\hat{I}_{s}(\tau)=U^{*} I_{s}(\tau) ; \hat{u}_{s}=b_{s} U^{*} u_{s} ; \Lambda(i, j)$ и $\Lambda(-i, j)$ - диагональные матрицы размерности $p \times p$, диагональные элементы $\lambda_{k}( \pm i, j)$ которых определяются формулами

$$
\begin{gathered}
\lambda_{k}(i, j)=\left(\lambda b_{i} \alpha_{j} / 4 q\right)\left(g\left(\gamma_{i j}^{0}\right)+(-1)^{k} g\left(\gamma_{i j}^{q}\right)+2 \sum_{l=1}^{q-1} g\left(\gamma_{i j}^{l}\right) \cos (\pi k l / q)\right), \\
\lambda_{k}(-i, j)=-\left(\lambda b_{i} \alpha_{j} / 4 q\right)\left(g\left(\pi-\gamma_{i j}^{q}\right)+(-1)^{k} g\left(\pi-\gamma_{i j}^{0}\right)+\right. \\
\left.+2 \sum_{l=1}^{q-l} g\left(\pi-\gamma_{i j}^{q-l}\right) \cos (\pi k l / q)\right), \quad i, j=1,2, \ldots, n ; k=1,2, \ldots, 2 q,
\end{gathered}
$$

а $k$-й элемент $\hat{u}_{s k}$ вектора $\hat{u}_{s}$ есть

$$
\begin{gathered}
\hat{u}_{i k}=\left(\lambda c_{0} b_{i} / 4 \pi \sqrt{2 q}\right)\left(g\left(\gamma_{i 0}^{0}\right)+(-1)^{k} g\left(\gamma_{i 0}^{q}\right)+2 \sum_{l=1}^{q-l} g\left(\gamma_{i 0}^{l}\right) \cos (\pi k l / q)\right), \\
\hat{u}_{-i k}=-\left(\lambda c_{0} b_{i} / 4 \pi \sqrt{2 q}\right)\left(g\left(\pi-\gamma_{i 0}^{q}\right)+(-1)^{k} g\left(\pi-\gamma_{i 0}^{0}\right)+\right. \\
\left.+2 \sum_{l=1}^{q-l} g\left(\pi-\gamma_{i 0}^{q-l}\right) \cos (\pi k l / q)\right), \quad k=1,2, \ldots, 2 q,
\end{gathered}
$$

$\cos \left(\gamma_{i j}^{l}\right)=\mu_{i} \mu_{j}+\sqrt{\left(1-\mu_{i}^{2}\right)\left(1-\mu_{j}^{2}\right)} \cos (l \pi / q), i=1,2, \ldots, n ; j=0,1, \ldots, n$.

Зная решение $\hat{I}_{s}(\tau)$ задачи $(30)$, можно легко определить решение $I_{s}(\tau)=\operatorname{Re}\left(U \hat{I}_{s}(\tau)\right)$ задачи $(20)$

$$
I_{s m}(\tau)=\operatorname{Re}\left(1 / \sqrt{p} \sum_{l=1}^{p} r_{l}^{m-1} \hat{I}_{s l}(\tau)\right)=(1 / \sqrt{2 q}) \sum_{l=1}^{2 q} \hat{I}_{s l}(\tau) \cos (\pi l(m-1) / q) .
$$




\section{6. Некоторые обсуждения}

Итак, решение уравнения переноса излучения (1)-(2) методом дискретных ординат сводится к решению системы дифференциальных уравнений (30). Матрицу коэффициентов $\hat{R}$ этой системы можно описать следующим образом: она состоит из элементов $\Lambda(i, j)$ и $\Lambda(-i, j), i, j=$ $=1,2, \ldots, n$, являющихся диагональными матрицами, и имеет размерность $2 n \times 2 n$. Это позволяет применять к задаче (30) без значительных изменений многие методы решения краевых задач как к системе размерности $2 n \times 2 n$, эффективно используя многопроцессорные машины при численной реализации.

Обозначим количество операций, необходимых для решения задачи $(30)$, через $k(n, p)$. Учитывая строение матрицы $\hat{R}$, легко получаем формулу

$$
k(n, p)=p k(n, 1) .
$$

Величину $k(n, 1)$ можно интерпретировать как количество операций, необходимых для решения системы дифференциальных уравнений с краевыми условиями размерности $2 n \times 2 n$. Эта величина зависит от конкретного метода.

Отметим еще одно свойство системы (30). Несмотря на то, что количество операций, необходимых для решения задачи $(30)$, есть $k(n, p)$, на окончательный результат будет оказывать влияние только $k(n, 1)$ ошибок округления результатов промежуточных вычислений.

\section{Л ИТ Е Р А Т У Р А}

1. М ар чук Г. И., Лебедев В. И., Численные методы в теорин переноса нейтронов, М., Атомиздат, 1971.

2. Ч а ндр а с ек а р С., Перенос лучистой энергии, М., ИЛ, 1953.

3. Х ейн ло А., В и й к Т., В кн.: Перенос излучения в несером слое и образование линий поглощения, Тарту, АН ЭССР, 1978, с. 37-54.

4. С оболе в В. В., Рассеяние света в атмосферах планет, М., «Наука», 1972.

5. Кн я зи хи н Ю., Уч. зап. Тартуск. ун-та, 500, 73-91 (1979).

6. Бе л м ан н Р., Введение в теорию матриц, М., «Наука», 1976.

7. Р и с с Ф., Сёкефальви-Надь Б., Лекции по функциональному анализу, М., «Мир», 1979.

Ннститут астрофизики и физики атмосферы Академии наук Эстонской ССР

Поступила вं редакцию 14/IX 1981

\section{J. KNJAZIHHIN}

\section{DISKREETSETE ORDINAATIDE MEETODI KASUTATAVUS KIIRGUSLEVI VORRANDI LAHENDAMISEKS HOMOGEENSE, TASAPARALLEELSE, ANISOTROOPSELT HAJUTAVA ATMOSFÄĂRI KORRAL. 1}

Kiirguslevi integraal-diferentsiaalvõrrand on lahendatud diskreetsete ordinaatide meetodil. On näidatud, et sobivalt valitud kvadratuurvalemi korral laguneb diferentsiaalvõrrandi koefitsientide maatriks omavahel kommuteeruvateks plokkideks, mida on lihtne unitaarmaatriksi abil diagonaalkujuks taandada. 


\section{INVESTIGATION OF THE DISCRETE ORDINATES SOLUTION OF THE RADIATIVE TRANSFER EQUATION IN THE HOMOGENEOUS, PLANE-PARALLEL, ANISOTROPICALLY SCATTERING ATMOSPHERE. 1}

The given paper presents the first part of the investigation of the discrete ordinates solution of the radiative transfer equation in the homogeneous, plane-parallel, anisotropically scattering atmosphere. The following variant of this method is considered the double integral which occurs in the integro-differential equation of the radiative transfer is replaced by the quadrature formulae. Due to the use of this discretisation, we can replace the radiative transfer equation (1) by a system of $2 n p$ linear differential equations (6).

Let the formula (5) be the rectangular formula. In this case the system of linear differential equations (6) can be transformed to the system of linear differential equations (30). It has the following structure - the matrix dimension of the coefficient of the system $(30)$ is of the order $2 n$ and its elements are $p \times p$ diagonal matrices. 